\title{
Magnitude of Bacteriological Etiology in Acute Encephalitis Syndrome: A Cross-Sectional Study
}

\author{
Prabhat Bhardwaj', Richa Tripathi \\ ${ }^{1}$ Assistant Professor, Department of Paediatrics, G S Medical College \& Hospital, Pilkhuwa, Uttar Pradesh, India, ${ }^{2}$ Medical Officer, District Hospital, District Hospital \\ Ghaziabad, Uttar Pradesh, India.
}

\section{Abstract}

Background: Meningoencephalitis is an acute purulent infection within the subarachnoid space, it is associated with a CNS inflammatory reaction i.e. inflammation of brain and parenchyma both that may result in decreased consciousness, seizures, raised intracranial pressure and stroke. The present study was aimed to know the magnitude of bacteria that making picture of meningoencephalitis in AES among the children. Subjects and Methods: Present cross-sectional study was conducted in Children aged 1-15 yrs of age suffering from AES admitted in B.R.D Medical College, Gorakhpur. The study subject (children aged 1-15yrs suffering from AES) have been investigated for AES and bacterial etiology and subsequent comparison is made on the basic of clinical features, CSF findings and other lab findings. We divided our study in two parts, in first on the basic of our inclusion criteria we enrolled the patient and on a preplanned clinical proforma we did detailed clinical examination and investigate them accordingly. Cases with suspected AES were studied for their clinical features, recovery patterns and other biochemical and microbiological parameters. After the patient was admitted in the hospital, CSF, serum and stool samples were collected and stored at appropriate temperature. The samples were collected by resident doctor. Results: Out of 160 cases 16 case show culture positive result and among them $\mathrm{Gm}$ +ve cocci was found in only 2 cases $(2.5 \%)$. Gram negative cocci in 10(6.25\%) cases and gram negative bacilli in 4 $(2.5 \%)$ cases. The most common presenting symptoms were fever in 16 (100) cases followed by vomiting in 12 (75.00), headache in 10 (62.55) a seizure was in 10 (62.5) cases that was generalized tonic type. The CSF examination was suggestive that the majority of cases of AES were due to viral encephalitis. And 10\% culture show positivity of bacteria in CSF. Meningeal sign like neck rigidity and kernign have significant association with bacterial culture positive result. Conclusion: The most common illness among AES patients observed in our study is NonBacterial but Japanese Encephalitis is still a problem in this region and it must not be neglected. Better diagnostic tests and standards to link an agent to encephalitis are needed in this field.

Keywords: Children, Culture positive, Gram negative cocci, Meningoencephalitis.

Corresponding Author: Dr Richa Tripathi, Medical Officer, District Hospital Ghaziabad, Uttar Pradesh, India.

Received: November 2019

Accepted: November 2019

\section{Introduction}

Acute encephalitis syndrome is a major health problem in north eastern Uttar Pradesh since 1978. ${ }^{[2]}$ The disease affects persons mainly from 7 districts under Gorakhpur and Basti division namely Gorakhpur, Basti, Deoria, Maharajganj, Santkabeer Nagar, Siddharth Nagar and Kushinagar and two adjoining district of Bihar namely Gopalganj and West Champaran. This is terai area bordering Nepal in the North and Bihar in the east. This region falls in tropical zone were extensive cultivation of rice during monsoon facilitate suitable breeding conditions for mosquitoes and other vectors. ${ }^{[4]}$

It affects thousands of patients presenting as epidemic mostly in post monsoon period. ${ }^{[3]}$ The epidemic of 2005 was one of the worst, ${ }^{[4]}$ with highest number of patients of this disease (about 5000) admitted in different institutions of the region. Out of which 1300 died. It drew the attention of international community as well. A massive immunization campaign using Chinese live attenuated strain SA-14-14-2 was launched in the year 2005-2006. ${ }^{[5]}$

The main cause of such epidemic has been an arthropod borne viral infection caused by Japanese encephalitis (JE) virus. In addition to Japanese encephalitis (8 to 10\%), other important etiological agents are bacteria (8 to 10\%) protozoa and some other viruses most probably enterovirus. ${ }^{[7-10]}$

So it is observed that acute encephalitis syndrome cases with multiple aetiologies are occurring in eastern UP9. Most of the cases are admitted in Nehru Hospital BRD medical college, Gorakhpur, Various agencies have been working to study the epidemiology, clinical feature and outcome of AES. Massive efforts as well have been made for the etiology1 of encephalitis in this region. Among all these cases of AES, there are cases having picture of meningitis like neck rigidity, kerning sign, other feature of raised intracranial tension, with or without focal neurological sign, whom we labelled as meningoencephalitis and on investigation it is found that having bacterial etiology. ${ }^{[10]}$ Meningoencephalitis is an acute purulent infection within 
the subarachnoid space, it is associated with a CNS inflammatory reaction i.e. inflammation of brain and parenchyma both that may result in decreased consciousness, seizures, raised intracranial pressure and stroke. The meninges, subarachnoid space and the brain parenchyma are all frequently involved in the inflammatory reaction (meningoencephalitis). It is the most common form of suppurative CNS infection with an annual incidence in the United States of $>2.5$ cases/100,000 population. ${ }^{[1,12]}$

The most common organism responsible is Streptococcus pneumoniae $(50 \%)$, followed by N. Meningitidis $(25 \%)$, group B streptococci (15\%), Listeria monocytogenes (10\%) and $\mathrm{H}$. Influenzae $(<10 \%){ }^{[10-12]}$

It can present as either an acute fulminant illness that progresses rapidly in a few hours or as a subacute infection that progressively worsens over several days. The classic triad of meningitis is fever, headache and nuchal rigidity. ${ }^{[10,12]}$ A decreased level of consciousness occurs in $>75 \%$ of patients and can vary from lethargy to coma. Nausea, vomiting and photophobia are also common complaints. Seizures are present in $20-40 \%$ of patients. Signs of raised ICT may be present like deteriorating or reduced level of consciousness, papilledema, dilated poorly reactive pupils, $6^{\text {th }}$ nerve palsies, decerebrate posturing, cerebral herniation and Cushing reflex. A diffuse erythematous maculopapular rash may be seen in meningococcemia. ${ }^{[13]}$

The present study was aimed to know the magnitude of bacteria that making picture of meningoencephalitis in AES among the children. An attempt was also made to study the differences in the various demographic, clinical features, secondary complication in these children and comparison of bacterial and non bacterial cases.

\section{Subjects and Methods}

Present cross-sectional study was conducted in Children aged 1-15 yrs of age suffering from AES admitted in B.R.D Medical College, Gorakhpur.

The study subject (children aged 1-15yrs suffering from AES) have been investigated for AES and bacterial etiology and subsequent comparison is made on the basic of clinical features, CSF findings and other lab findings.

The study subject were identified at the time of admission in Pediatrics ward of B.R.D. Medical College based on clinical criteria (inclusion and exclusion criteria) defined by W.H.O. FOR AES patients.

\section{Inclusion Criteria}

- All children aged 1-15yrs presenting with

- Acute onset (14days ) of fever (>100F) with seizures lasting>60 minutes.

- Acute onset (14days ) of fever (>100F) with focal neurological deficit.

- Acute onset (14days ) of fever (>100F) and a change in mental status (including symptoms such as confusion, disorientation, coma, or inability to talk)
- Acute onset (14days ) of fever (>100F) with abnormal behavior or an increase in irritability somnolence greater than that seen with usual febrile illness.

- Acute onset of fever with headache, vomiting with altered sensorium or irritability

\section{Exclusion Criteria}

- A febrile with seizures and neurological deficit.

- Simple febrile seizures (seizure<15 min, unconciuosness $<1 \mathrm{hr}$ in a child, 6m-5yrs, with normal CSF)

- Acute encephalopathy with documented metabolic derangement, presenting with no headache, no FND, with near normal CSF and EEG showing diffuse slowing on investigation

- Microcephaly, previous mental retardation, cerebral palsy.

We divided our study in two parts, in first on the basic of our inclusion criteria we enrolled the patient and on a preplanned clinical proforma we did detailed clinical examination and investigate them accordingly. As per objective and in second part we observed all AES cases and compare culture positive case with culture negative cases. All suspected case of acute encephalitis syndrome are examined clinically and investigated as per objective.

Primary objective was to the know the magnitude of bacterial etiology so we take all those case positive in which CSF culture is positive for bacteria. So our primary investigation is CSF so lumber puncture is done under all aseptic precaution on the day of admission of patient and CSF withdrawn. This CSF divided into 3 parts, in which one part 20 microlitre for CSF culture, 500microlitre for cytobiochemistry and the 3rd part of CSF samples was examined in NIV laboratory for the detection of viral causes (arboviruses, herpesviruses, enteroviruses).

Among all those CSF in which culture report and gram staining test show positive result these cases correlated clinically with culture negative cases.

The clinical status of all those culture positive cases was further evaluated statically for any correlation.

Culture positive case: Any case that is enrolled and undergone CSF examination and their bacterial culture show positive result taken as positive case ireescpective of meningeal sign and CSF cytobiochemical change. Other investigation done to isolate the viral etiology.

Following culture media available for isolating organism-

1) Blood agar and chocolate agar; plates incubated at $37^{\circ} \mathrm{C}$ for 72 hours in $5-10 \% \mathrm{CO}$ t2.

2) Mac Conkey's agar, incubated at $37^{\circ} \mathrm{C}$ for 72 hours.

Sample size: Calculated by using following formulae $\mathrm{N}=\mathrm{z} 2 \mathrm{pq} / \mathrm{d} 2$

Past record show that approx 2000 AES cases are admitted in BRD MEDICAL COLLEGE each year. So to cover a 
sample size of 175 the sampling interval estimated was 11. The AES no allotted in line listing of cases admitted at BRD MEDICAL COLLEGE was used for sampling.

The first case was chosen using a random no. generated through computer software and then every 11th case was used for sampling till desired sample size of 175 was attained.

The clinical history included the duration of fever, headache, vomiting, altered sensorium, seizures, paralysis, cough, breathlessness, chest and abdominal pain, diarrhoea, swelling of body and gastrointestinal bleeding. The examination included general condition of patient, pulse, blood pressure, respiratory rate, temperature, pallor, icterus, cyanosis, clubbing, edema, lymphadenopathy and state of hydration. A detailed systemic examination was carried out with special emphasis, on the state of higher centres, level of consciousness, signs of meningeal irritation like neck rigidity ,kenign sign, involvement of cranial nerves, sensory, motor examinations, superficial and deep tendon reflexes, cerebellar signs, involuntary movements, gait of the patients and fundus examination.

The cardiovascular system examination included tachycardia, peripheral circulatory failure, gallop rhythm, features of congestive heart failure, heart murmurs and heart sounds (muffled or loud). Abdominal examination included hepatomegaly, splenomegaly, hepatosplenomegaly and ascites. Respiratory system examination includes types of breath sounds, adventitious breath sounds (crepts/rhonchi) and respiratory failure.

Cases with suspected AES were studied for their clinical features, recovery patterns and other biochemical and microbiological parameters. After the patient was admitted in the hospital, CSF, serum and stool samples were collected and stored at appropriate temperature. The samples were collected by resident doctor.

\section{1) Blood investigation-}

Haemoglobin, total leucocyte count, differential leucocyte count, platelet count, random blood sugar, general blood picture, renal function tests, liver function tests, serum electrolytes.

\section{2) Urine examination-}

Routine and microscopic examination.

3) CSF examination-

Complete cytobiochemical examination, gram's staining, immunological examination and culture.

4) Chest X-ray-

To find any cardiomegaly or pulmonary congestion.

5) ECG-

To look for myocarditis or heart failure.

6) IgM ELISA Detection-

For Japanese Encephalitis virus in serum and CSF. Detection of IgM antibodies against JE virus from serum and CSF.

7) CT scan and MRI Brain

8) Real Time RT PCR Assay for Quantitation of the viral
RNA from the specimens

9) CSF culture for bacteria is Gold standard tool for isolating bacteria.

Following culture media available for isolating organism

1) Blood agar and chocolate agar; plates incubated at $37^{\circ} \mathrm{C}$ for 72 hours in $5-10 \% \mathrm{CO} 2$.

2) Mac Conkey’s agar, incubated at $37^{\circ} \mathrm{C}$ for 72 hours.

\section{Statistical analysis:}

The recorded data was compiled and entered in a spreadsheet computer program (Microsoft Excel 2007) and then exported to data editor page of SPSS version 15 (SPSS Inc., Chicago, Illinois, USA).

Descriptive statistics included computation of percentages, means and standard deviations. For all tests, confidence level and level of significance were set at $95 \%$ and $5 \%$ respectively.

\section{Results}

A total of 175 patients were enrolled into the study. Of these 15 patients were excluded from the study either due to insufficient data, non cooperative patients and patients in which convalescent samples could not be collected. Thus, out of 175 patients 160 patients were subjected to detailed clinical examination and investigations They were admitted in the pediatric wards of the Nehru hospital, attached to the B.R.D. Medical college Gorakhpur.

During this period, the total hospital admissions were 45475 and total AES patients were 2517 thus AES constitutes $(5.5 \%)$ of total hospital admissions. Among the total AES patients, 2338 were admitted in the pedia department thus it constitute $5.1 \%$ of total hospital admissions.

Out of 160 cases, Non-Bacterial (culture negative) is 144 (90\%), Bacterial positivity (culture positive) found in 16 (10\%) case.

Out of 160 cases, non-bacterial (culture negative) is 144 (90\%), Bacterial positivity (culture positive) found in 16 $(10 \%)$ case.

Out of 160 cases 16 case show culture positive result and among them $\mathrm{Gm}$ +ve cocci was found in only 2 cases $(2.5 \%)$. Gram negative cocci in $10(6.25 \%)$ cases and gram negative bacilli in $4(2.5 \%)$ cases. The most common presenting symptoms were fever in 16 (100) cases followed by vomiting in 12 (75.00), headache in 10 (62.55) a seizure was in 10 (62.5) cases that was generalized tonic type. 10 cases out of 16 cases have GCS between 7 to 10 and 4 cases show GCS $<7$.

In Culture positive cases most common finding was plantar extensor 12/16 and then sign of meningeal irritation10/16,brisk dtr in 5/16 cases, sign of raised ict like papilloedema in fundus was present in 5/16 cases, hemiparesis was found in only 1 case. CSF pleocytosis was seen in 14 cases $(87.5 \%)$, but in majority of them the TLC 
$>100$ \{case $(62.5 \%)\}$. The TLC between 6 to 100 was found in only 4 cases $(25 \%)$. The Protein less than $60 \mathrm{mg} / \mathrm{dl}$ was present in 5 cases $(31.25 \%)$ and more than 60 in $11(68.75)$. Sugar was decreased in 8 cases $(50 \%)$ and increased in 8 $(50 \%)$ cases. In culture positive cases SGPT was raised in $10 / 16$ (62.5) leucocytosis was found in 8/16 (62.5) and creatinine $1 / 16$ (6.25). Among culture positive case $25 \%$ cases show meningeal enhancement 1 case show cortical hypodensity and 2 case show hydrocephalus. In culture positive cases hepatomegaly was found in 3 cases and spleen in one case.

The most common presenting symptoms were fever (100\%) and in both groups, Temperature $\geq 100 \circ \mathrm{F}$ was predominant feature in both group, Vomiting in 38 (26.38\%) in nonbacterial and $12(75.00 \%)$ in bacterial. Headache were present in and $30(20.83 \%)$ of Nonbacterial and 10 (62.50) in bacterial case, both headache and vomiting was statistically significant $(\mathrm{p}<.05)$ in both group.

Seizure present in 103 cases $(64.37 \%)$ of non bacterial and 10 (62.5) of bacterial case then altered sensorium in 82(56.94) case of non bacterial and 10 (62.5) of bacterial case [Table1]

The commonest CNS finding was planter extensor $\{114$ $(79.16 \%)$ in Non bacterial and $12(75 \%)$ in bacterial\}But that difference is statistical no significant next common being signs of meningeal irritation $10\{62.5 \%$ ) in bacterial and in nonbacterial case $5(3.47 \%)$ case that is statistically highly significant, brisk dtr $103(71.52 \%)$ in nonbacterial and in bacterial 5 cases (31.25), hypertonia 105 (72.91) case in nonbacterial and 3 cases (18.75) and are statistical significance, Fundus in $14(9.7 \%)$ case of non bacterial and $5(31.25 \%)$ case of bacterial and papillary changes in nonbacterial case is $22(15.27 \%)$ and in bacterial case it is $6(37.5 \%)$ are statistically significant, hemiparesis were not statistically significant in both group [Table2]. There e was no statistical significance difference between the two groups [Table 2].

In both group hepatomegaly was significantly associated, no other finding show statistical significance difference between the two groups [Table 3]. Higher count in both group was highly statistically significant and there is significant association between increased total leucocyte count and bacterial culture positivity i.e. so increased total leucocyte count have more chance of positivity of bacterial culture but other finding like haemoglobin, and serum creatinine and SGPT between two groups have no significant association [Table 4].

CSF pleocytosis was found in $14(87.50 \%)$ of bacterial cases and $(65.27 \%)$ of Non bacterial however cells were less than 100 in majority in nonbacterial groups. Polymorphic dominance was seen in $10(62.50 \%)$ cases of bacterial, while it was $27(18.75 \%)$ for Non bacterial. Both are statistically significant. Raised protein $>60$ was found in majority of cases in both groups, not statistically significant, Sugar was in 20 case of nonbacterial reduced $(13.88 \%)$ and $(50 \%)$ of bacterial that was statistically significant difference in CSF findings [Table 5].

The most common CT scan finding in both group was meningeal enhancement but there was no significant difference in CT scan findings between two group. There was no significant difference in mortality between two groups. It is observed that culture positivity is more in less than 5 year of age group and it also show significant association with culture negative case.

A total of 51 cases $(31.9 \%)$ belonged to less than 7 yrs of age, suggesting the disease had more incidences in younger children population. Male: Female ratio was 1.2: 1. Although, the cases were seen throughout the year but the incidence peaked to $58(36.25 \%)$ in the month of September and $50(31.25 \%)$ in the month of October, suggesting the seasonal occurrence of the disease .

Patients of AES came here from Gorakhpur and its neighbouring districts as well as Bihar and Nepal. The largest no. of cases came from Gorakhpur 48 (30\%), followed by, Kushinagar 32 (20\%), Deoria 27(16.8\%) and Maharajganj 9 (5.62\%). The most common presenting symptoms were fever in 160 cases, seizure in 113cases and altered sensorium in 92 cases each $(57.5 \%)$, followed by headache in $40(25 \%)$ and vomiting in $50(31.25 \%)$ [Table 6]. Pyrexia was predominant feature followed by tachypnea in $72(45 \%)$ tachycardia in $118(73.75 \%)$ and pallor in. Icterus in $30(18.75 \%)$ and edema $26(16.25 \%)$ were present [Table 7]. At the time of admission GCS was between 7-10 in $91(56.87 \%)$ cases in the GCS between 3-6, found in $52(32.50 \%)$ cases and $\geq 10$ was found in 17 $(10.62 \%)$ cases. The mean GCS at the time of admission was 8.95 .

The most common CNS examination finding was planter extensor, found in 126 cases $(78.75 \%)$, signs of meningeal irritation in $15(9.30 \%)$, brisk DTR in $108(67.5 \%)$ and papilledema in 19(11.87\%). Extrapyramidal and cerebellar signs were found in only $8(5 \%)$ and $7(4.37 \%)$ cases respectively, while hemiparesis was found in only 2 (1.25\%).Hepatomegaly was the most common finding of abdominal examination found in 83 cases $(51.87 \%)$, splenomegaly in $39(24.37 \%)$ followed by hepatosplenomegaly and in 39 (24.37\%). Adventitious sounds (crepts/rhonchi) were present in 30 cases $(18.75 \%)$, followed by respiratory failure in $6(3.75 \%)$, muffled heart sound was the most common cardiac abnormality found in 53 cases $(33.12 \%)$, followed by gallop rhythm in 28 (17.50) cases. The hemoglobin of the cases was between $9-12 \mathrm{gm} / \mathrm{dl}$ in majority $\{135$ cases $(84.37 \%)\}$, followed by $\geq 12$ in 12 $(7.5 \%)$.The mean haemoglobin of the cases was 11.13 $\mathrm{gm} / \mathrm{dl}$. Leucocytosis was found in 38 cases $(23.75 . \%)$. SGPT was raised in 119 cases $(74.37 \%)$. Serum creatinine was raised in 10 cases $(6.25 \%)$ [Table 8 ].

CSF pleocytosis was seen in 108 cases $(65.62 \%)$, but in majority of them the TLC was between 6 to 100 \{68 case $(42.5 \%)\}$.The TLC more than 100 was found in 40 cases 
$(25 \%)$. The Protein between $60-100 \mathrm{mg} / \mathrm{dl}$ was present in 100 cases $(62.5 \%)$ and more than 100 in only $10(6.25)$. Sugar was decreased in 28 cases $(17.5 \%)$ and normal in rest of them.

Among culture positive result $\mathrm{Gm}$ +ve cocci was found in only 2 cases $(1.25 \%)$. Gram negative cocci in 10 (6.25) cases and gram negative bacilli in $4(2.5 \%)$ cases. The CSF examination was suggestive of that the majority of the cases were of Acute viral encephalitis.

The most common CT scan finding was meningeal enhancement seen in 16 cases $(10 \%)$, followed by cortical hypodensities in $6(3 \%)$ and hydrocephalus. The most common cause of AES was (Non-Bacterial) responsible for 144cases (90\%), and Acute bacterial meningitis was responsible for $16(10 \%)$ case.

\section{Table 1: Showing Presenting Symptoms of the Cases}

\begin{tabular}{|l|l|l|l|}
\hline Symptoms & No. of CASES (non bacterial) (\%) & No. of CASES (bacterial) (\%) & 'p' value \\
\hline Fever & $144(100.0)$ & $16(100.0)$ & $<0.001$ \\
Headache & $30(20.83)$ & $10(62.50)$ & 0.002 \\
Vomitting & $38(26.38)$ & $12(75.00)$ & 0.002 \\
Altered sensorium & $82(56.94)$ & $10(62.50)$ & 0.669 \\
Seizures & $103(64.37)$ & $10(62.50)$ & 0.451 \\
Paralysis & $01(00.60)$ & $01(06.25)$ & $0.476^{*}$ \\
\hline
\end{tabular}

Figures Showing No (\%) ( $\mathrm{P}^{*}=$ Fisher Exact Test)

Table 2: Showing CNS Examination Findings of Bacterial and Non-Bacterial Cases

\begin{tabular}{|l|l|l|l|}
\hline CNS examination findings & No. of cases (non bacterial) (\%) & No. of cases (bacterial) (\%) & 'p' value \\
\hline Signs of meningeal irritation & $05(03.47)$ & $10(62.50)$ & $<0.001$ \\
Cranial nerve palsies & $09(06.25)$ & $02(12.50)$ & 0.676 \\
Pupil (Dilated and sluggish reacting) & $22(15.27)$ & $06(37.50)$ & 0.020 \\
Fundus (Papilledema) & $14(09.70)$ & $05(31.25)$ & 0.034 \\
Hypertonia & $105(72.91)$ & $03(18.75)$ & $<0.001$ \\
Hemiparesis & $01(00.60)$ & $01(06.25)$ & 0.476 \\
Brisk DTR & $103(71.52)$ & $05(31.25)$ & 0.002 \\
Plantar extensor & $114(79.16)$ & $12(75.00)$ & 0.948 \\
\hline Figures Showing No (\%) (P*=Fisher Exact Test)
\end{tabular}

Table 3: Showing Findings of Other Systemic Examination of Cases

\begin{tabular}{|c|c|c|c|}
\hline & No. of CASES (non bacterial) $(n=144)(\%)$ & No. of CASES (bacterial) $(\mathrm{n}=16)$ & 'p' value \\
\hline \multicolumn{4}{|l|}{ Abdominal examination } \\
\hline Hepatomegaly & $80(55.50)$ & $03(18.75)$ & 0.011 \\
\hline Splenomegaly & $37(25.19)$ & $02(12.50)$ & 0.390 \\
\hline Hepatosplenomegaly & $37(25.19)$ & $02(12.50)$ & 0.390 \\
\hline \multicolumn{4}{|l|}{ Respiratory examination } \\
\hline Adventitious sounds(crepts/rhonchi) & $27(18.75)$ & $03(18.75)$ & 0.735 \\
\hline Respiratory failure & $05(03.47)$ & $01(06.25)$ & 0.874 \\
\hline \multicolumn{4}{|l|}{ CVS examination } \\
\hline Raised JVP & $05(03.40)$ & $01(06.25)$ & 0.874 \\
\hline Muffled heart sounds & $50(34.72)$ & $03(18.75)$ & 0.313 \\
\hline Gallop rhythm & $25(17.36)$ & $03(18.75)$ & 0.835 \\
\hline Murmur & $02(01.30)$ & $00(00.00)$ & 0.476 \\
\hline
\end{tabular}

Figures Showing No (\%) $\left(\mathrm{P}^{*}=\right.$ Fisher Exact Test)

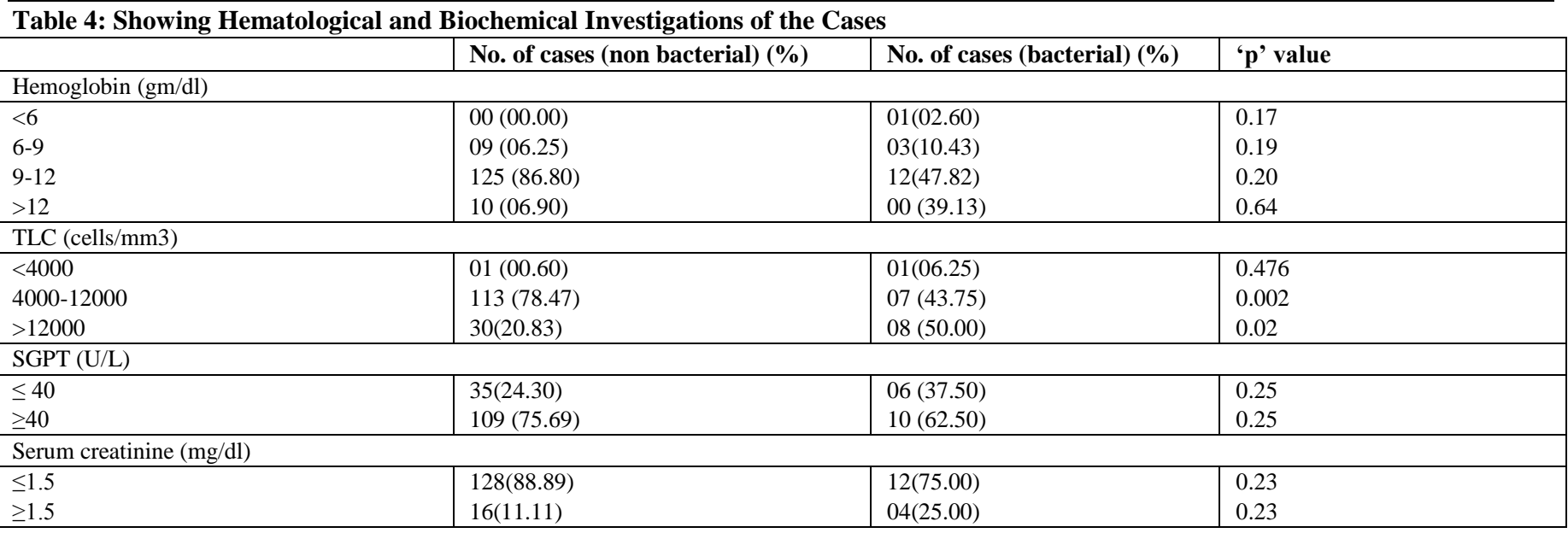

Figures Showing No $(\%)\left(\mathrm{P}^{*}=\right.$ Fisher Exact Test $)$ 


\begin{tabular}{|c|c|c|}
\hline No. of cases (non bacterial) (\%) & No. of cases (bacterial) (\%) & 'p' value \\
\hline $50(34.72)$ & $02(12.50)$ & 0.12 \\
\hline $30(20.83)$ & $10(62.50)$ & 0.002 \\
\hline \multicolumn{3}{|c|}{ DLC (cells/mm3) } \\
\hline $117(81.25)$ & $06(37.50)$ & 0.001 \\
\hline $45(31.25)$ & $05(31.25)$ & 1.00 \\
\hline $99(68.75)$ & $11(68.75)$ & 1.00 \\
\hline \multicolumn{3}{|c|}{ Sugar $(\mathrm{mg} / \mathrm{dl})$} \\
\hline $20(13.88)$ & $08(50.00)$ & 0.003 \\
\hline $124(86.11)$ & $08(50.00)$ & 0.003 \\
\hline
\end{tabular}

Figures Showing No $(\%)\left(\mathrm{P}^{*}=\right.$ Fisher Exact Test)

\begin{tabular}{|l|l|}
\hline Table: 6 Clinical Features of the Cases \\
\hline Symptoms & No. of cases (\%) \\
\hline Fever & $160(100.0)$ \\
Headache & $40(25.00)$ \\
Vomiting & $50(31.25)$ \\
Altered sensorium & $92(57.50)$ \\
Seizures & $113(70.63)$ \\
Rashes & $02(12.50)$ \\
Breathlessness & $31(19.40)$ \\
Abdominal pain & $05(03.12)$ \\
Loose stools & $31(19.37)$ \\
Swelling of body & $23(14.37)$ \\
Cough & $05(03.12)$ \\
\hline
\end{tabular}

Table 7: General Examination Findings of all AES Cases

\begin{tabular}{|l|l|}
\hline General examination findings & No. of cases $(\%)$ \\
\hline Temperature $(\geq 100 \circ \mathrm{F})$ & $160(100.0)$ \\
Tachycardia & $118(73.75)$ \\
Tachypnea & $72(45.00)$ \\
Pallor & $33(16.50)$ \\
Peripheral circulatory failure & $08(04.00)$ \\
Icterus & $30(18.75)$ \\
Edema & $26(16.25)$ \\
Lymphadenopathy & $04(02.00)$ \\
Cyanosis & $04(02.50)$ \\
Clubbing & $02(01.20)$ \\
\hline
\end{tabular}

Table 8: Hematological And Biochemical Investigations Of The Aes Cases

\begin{tabular}{|c|c|}
\hline & No. of cases (\%) \\
\hline \multicolumn{2}{|c|}{ Hemoglobin $(\mathrm{gm} / \mathrm{dl})$} \\
\hline$\leq 6$ & $01(00.62)$ \\
\hline $6-9$ & $12(07.50)$ \\
\hline $9-2$ & $137(85.62)$ \\
\hline$\geq 12$ & $10(06.25)$ \\
\hline \multicolumn{2}{|c|}{ TLC (cells/mm3) } \\
\hline$\leq 4000$ & $02(01.25)$ \\
\hline $4000-12000$ & $120(75.00)$ \\
\hline$\geq 12000$ & $38(23.75)$ \\
\hline \multicolumn{2}{|l|}{ SGPT (U/L) } \\
\hline$\leq 40$ & $41(25.63)$ \\
\hline$\geq 40$ & $119(74.37)$ \\
\hline \multicolumn{2}{|l|}{ SGOT(U/L) } \\
\hline$<40$ & $42(26.25)$ \\
\hline$>40$ & $120(74.75)$ \\
\hline \multicolumn{2}{|c|}{ Serum creatinine $(\mathrm{mg} / \mathrm{dl})$} \\
\hline$\leq 1.2$ & $140(87.50)$ \\
\hline$\geq 1.2$ & $20(12.50)$ \\
\hline
\end{tabular}

\section{Discussion}

The present study was carried out in the Department of Pediatrics, B.R.D. Medical College, Gorakhpur with the laboratory support of the National Institute of Virology, Gorakhpur Unit and Pune. The period of study was from August 2011 to Nov 2012.

North eastern Uttar Pradesh (U.P.) draws national and international attention due to continuing epidemic of acute viral encephalitis and its changing picture temporally. It has a wide socio-political implication. Most of the patients with acute encephalitis syndrome (AES) are children, though a significant number are adults as well. Several epidemics of AES recurring since 1978 have drawn massive public and political attention, despite all the efforts, the epidemics are regularly occurring with heavy mortality and morbidity. The most severe epidemic so far has been of 2005 with lot of hue and cry in the public. ${ }^{[4]}$ This forced a massive vaccination in the whole of north eastern UP and surprisingly, the no. of patients of AES still kept on pouring. Significantly it was observed that the JE positivity declined continuously and a large no. of patients (70-80\%) did not show JE infection.

Majority of cases etiology was not defined and it was assumed that the some bacteria were responsible for these encephalitis patients. However despite all the efforts bacterial positivity could not be established hence detailed analysis of clinical profile of these patients along with necessary lab investigation was done to know the how much $\%$ of AES cases are of bacterial in origin.

Mostly the patients with AES belong to pediatric age group and few studies have being done about the magnitude of bacterial etiology in these patients. Unfortunately no many studies are available on this. This is the first study where patients with AES have being studied. Unfortunately since no similar studies have been done in other parts of the country, where clinical spectrum is discussed so our observation on clinical background is not comparable.

One study named "Microbial Study of Meningitis and Encephalitis Cases" 14 of S. Selim et al done in microbiology department of alexandaria showing Out of 79 clinically diagnosed encephalitis cases, 10 (12.7\%) cases 
were due to bacterial causes, and $6(7.6 \%)$ were due to viral causes. Various thesis and literature work done in previous year of our department showing $8.5 \%$ case positivity .our study was aimed to know the magnitude of bacterial etiology, we will also discuss and compare clinical feature of all those case that show positive blood culture and other case that show culture negative result. So we made two group, one group that show culture positive result (BACTERIAL) and other group that show culture negative (NONBACTERIAL) result.

Among all case of AES the most common cause of AES was acute viral (Nonbacterial) encephalitis (90\%) of the cases. It was followed by Acute bacterial meningitis was responsible for $10 \%$ cases. Among culture positive cases $\mathrm{Gm}+\mathrm{ve}$ cocci was found in only $1.25 \%$, while gm -ve cocci in $6.25 \%$ and gram negative bacilli were found in $2.5 \%$ case

In our study to know magnitude of bacterial etiology we took culture positive as positive case irrespective of clinical sign so we got $10 \%$ case as culture positive case while one study named Microbial Study of Meningitis and Encephalitis Cases" of S. Selim et al, ${ }^{[14]}$ show $12 \%$ case bacterial in encephalitis one reason of more magnitude can be explained as they also used clinical criteria ie meningeal sign and significant cytobiochemical change so \% increased in term of magnitude.

One other study show $20 \%$ case bacterial positive higher $\%$ may be due to CSF cytobiochemistry change that support bacterial picture taken as positive case while in our study we take only those case positive where culture is positive. ${ }^{[15]}$

Age is significantly associated between culture positive and culture negative cases as lesser age group have more chance of positivity of bacteria in their culture than more age group. Male: Female ratio was 1.3: 1. A male predominance was noted in our study, the reason of this is not clear, but probably social customs of male preference is responsible for this discrepancy. Although, the cases were seen throughout the year but the incidence peaked to $36.25 \%$ in the month of September and $31.25 \%$ in the month of October, suggesting the seasonal occurrence of the disease. The peak incidence of the disease was from August to October i.e post monsoon period. The incidence of arboviral and enterovirus infections as well as malaria increases in this period.

Patients of AES came here mainly from Gorakhpur and its neighbouring districts as well as Bihar and Nepal. The largest no. of cases came from Gorakhpur 24.5\%, followed by, Kushinagar 20\% Deoria $16.87 \%$ and Maharajganj $5.62 \%$.

The most common presenting symptoms in all AES cases was fever and altered sensorium in $57.5 \% \%$ cases, and Seizures were present in $70.63 \%$ cases, headache in $25 \%$ and vomiting in $31.25 \%$, loose stools in $19.37 \%$, breathlessness in $19.4 \%$, swelling over body in $14.37 \%$ and abdominal pain in $3.12 \%$. The clinical features that showed statistically significant difference between the two groups one that show positive CSF culture and other show negative, were headache $(62.5 \%)$ and vomiting $(75 \%)$ (P-value $<0.05$

The most common CNS examination finding among all case of AES was plantar extensor, found in $78.75 \%$, followed by brisk DTR in $67.5 \%$, signs of meningeal irritation in $9.30 \%$, and papilledema in $11.87 \%$.

In bacterial case after extensor plantar, sign of meningeal irritation was most common finding, fundus changes also predominant, meningeal sign (kerning sign, neck rigidity), fundus and papillary reaction show significant difference $(\mathrm{p}<.05)$ between two groups.

Presence of meningeal sign like neck rigidity kerning sign show significant association with culture positive result of bacteria it mean that there are more chance of bacterial positivity of CSF culture in those case where meningeal sign are found, our result compare with available text that show case where meningeal sign are found, more likely to be bacterial positive case. Sign of raised ICT i.e. papilloedema and papillary reaction are more common in those case where culture is positive for bacteria and it is significantly associated with nonbacterial (culture negative) case. Hepatomegaly was the most common finding of abdominal examination found in $(51.87 \%)$ of all AES cases Table-IV/8, in bacterial case $(18.75 \%)$ and nonbacterial $(55.5 \%)$ followed by hepatosplenomegaly in $(24.37 \%)$, in bacterial case $(12.5 \%)$ and non-bacterial case $(25.19 \%)$. (TableIII/4).Adventitious sounds (crepts/ rhonchi) were present in $18.75 \%$ cases. The more number of patients with adventitious sounds may be due to higher incidence of aspiration pneumonitis in AES patients. No other clinical finding showed statistically significant difference between the two group, except hepatomegaly it means systemic association also found in culture positive case like nonbacterial case (Culture negative case) but nonbacterial etiology have more chances of systemic involvement. There was no statistical significant difference $(\mathrm{p}>0.05)$ between the two groups regarding GCS.

The haemoglobin of the all AES cases was between 9-12 $\mathrm{gm} / \mathrm{dl}$ in majority $(85.62 \%)$, followed by 12 in $6.25 \%$. The mean haemoglobin of the cases was $11.13 \mathrm{gm} / \mathrm{dl}$. Leucocytosis was found in $23.75 \%$. SGPT was raised in $74.37 \%$ of cases. Serum creatinine was raised in $12.5 \%$. Deranged liver and renal functions may be due to multiorgan involvement due to enteroviruses. ${ }^{[16]}$

In comparison of bacterial and nonbacterial there is no statistically significant difference except leucocytosis that show bacterial culture positivity is more in those case where leucocytosis is more $(\mathrm{p}<.05)$ Generally it is observed that bacterial meningitis have increased leucocyte count in our study also support it. ${ }^{[17]}$

CSF pleocytosis was found in $14(87.50 \%)$ of bacterial cases and $94(65.27 \%)$ of Non-bacterial, Polymorphic dominance was seen in $10(62.50 \%)$ cases of bacterial, while it was 27 (18.75\%) for Non bacterial. Both are statistically significant in each group, it mean in non bacterial case it is lymphocytic 
predominance that dominant and in bacterial case polymormhic predominance dominant. In comparison of CSF count it is observed that those result that show culture positive result found to be having more cells in CSF comparison of culture negative result that show statistically significant difference in CSF findings.

Raised protein $>60$ was found in majority of cases in both groups that is statistically not significant, $\mathrm{p}>.05$ (TableIII/) explained in such a way in viral encephalitis or bacterial meningitis generally protein raised so in our study increased protein not make a difference on culture positive result. Sugar was reduced in 20 case of nonbacterial $(13.88 \%)$ and $8(50 \%)$ of bacterial that show statistically significant difference in CSF findings it mean sugar is decreased more in those case that show positive culture result. ${ }^{[18]}$ In one study comparison of the CSF laboratory values indicates that patients who had an infectious agent diagnosed had a higher CSF WBC count than did patients who had a noninfectious agent diagnosed (median CSF WBC count, 53.5 vs. 9.5 cells/mm3; P ! .001). However, the difference in CSF protein levels was not significant (median level, 71.0vs. 67.0 $\mathrm{mg} / \mathrm{dL}$ ), that also support our finding.

Among all cases of AES the CSF pleocytosis was seen in 108 case, $(65.62 \%)$, but in majority of them the TLC was between $6-100,68$ cases $(42.5 \%)$. The TLC more than 100 was found in $25 \% \%$ cases. In maximum patients the protein was between $60-100 \mathrm{mg} / \mathrm{dl}(62.50 \%)$. Sugar was decreased in 28 cases $17.5 \%$ and normal in rest of them.

$\mathrm{Gm}+\mathrm{ve}$ cocci was found in only $1.25 \%$, while gm -ve cocci in $6.25 \%$ and andgram negative bacilli were found in $2.5 \%$ cases. The CSF examination was suggestive of that the majority of the cases were of Acute viral encephalitis (and $10 \%$ case show bacterial culture positive result.

The most common CT scan finding was meningeal enhancement seen in $10 \%$ cases, followed by cortical hypodensities in $3.75 \%$ and hydrocephalus in $2.5 \%$. The most common CT scan finding in both cases was meningeal enhancement in bacterial 25\% case and non bacterial 8.3\% case. But there was no significant difference in CT scan findings between two groups.

The most common cause of AES was Non bacterial (90\%) of the cases. Acute bacterial meningitis was responsible for $10 \%$ cases,among which $\mathrm{Gm}+\mathrm{ve}$ cocci was found in only $1.25 \%$, while gm -ve cocci in $6.25 \%$ and andgram negative bacilli were found in $2.5 \%$ case among all positive CSF culture. Clearly this shows that our efforts to control AES has to be in a different direction than controlling only nonbacterial case, this calls for the separate epidemiologic studies to find out the exact etiological agent for the AES and thus planning the preventive measures.

\section{Conclusion}

The most common illness among AES patients observed in our study is Non-Bacterial but Japanese Encephalitis is still a problem in this region and it must not be neglected. Other main causes of acute encephalitis syndrome are acute bacterial meningitis (10\% bacterial positive) should never be forgotten. As major etiological agent of bacterial meningitis is vaccine preventable organism so vaccine preventable disease i.e. Japanese encephalitis, H. influenjae meningitis, meningococcal meningitis and pneumococcal meningitis by je vaccine, hib vaccine, meningococcal vaccine and pneumococcal vaccine should be controlled. As in other infections, the effective inactivation and disposal of sewage, high standard of personal hygiene, and control of files are necessary prophylactic requirements. General cleanliness, frequent hand washing in soap and water, avoidance of sharing towels, and the avoidance of contaminated water are important.

Any outbreaks of meningoencephalitis have never been reported in India and any epidemic has never been reported anywhere in the world. Although epidemic of meningococcal meningitis has occurred but no such study are available that show associtation of meningoencephalitis and their outbreak, so a surveillance system that include meningoencephalitis and viral encephalitis cases for such infection should be developed which should monitor the trends of such infection and further studies should be conducted to ascertain the clinical profile and epidemiological course of the disease, so that standard protocols can be developed and such patients are managed effectively.

Despite a concerted effort to diagnose cases with comprehensive testing for the expected cause of encephalitis, the etiologies of more than $50 \%$ of the cases remains elusive. Better diagnostic tests and standards to link an agent to encephalitis are needed in this field. Given the broad spectrum of agents identified and the overlap in epidemiological and clinical spectrum among cases with unknown etiologies, we suspect that unidentified infectious causes will probably include both known and yet to be established causes of encephalitis. New strategies for pathogen identification and continued analysis of exposures and clinical features should also help us improve our ability to diagnose, treat and prevent encephalitis.

\section{References}

1. Solomon T, Thao TT, Lewthwaite P, Ooi MH, Kneen R, Dung RN et al A Cohort Study to Assess the New WHO Japanese Enceph. Surveill Stand Bull WHO 2008; 86:178-86.

2. Mathur A, Chaturvedi UC, Tandon HO, Agarwal AK, Mathur GP, Nag D, et al. Japanese encephalitis in Uttar Pradesh, India during 1978. Indian J Med Res 1982; 75: 161-169.

3. Rodrigues FM. Epidemiology of Japanese encephalitis in India. National Conference on Japanese encephalitis. Indian J Med Res 1984; (suppl): 1-9.

4. Parida M, Dash PK, Tripathi NK, Ambuj, Sannarangaiah S, Saxena P, et al. Japanese Encephalitis Outbreak, India, 2005. Emerg Infect Dis 2006; 12: $1427-1430$.

5. Halstead SB, Jacobson J. Japanese encephalitis vaccines. In: Plotkin SA, Orenstein WA, Offit PA, editors. Vaccines. 5th ed. Philadelphia: 


\section{Bhardwaj d Sripathi; Magnitude of Bacterialagical Etialagy in Acute Encephalitis Syndrame}

Elsevier; 2008. pp. 311-52.

6. Kabilan L, Rajendran R, Arunachalam N, Ramesh S, srinivasan S, Philip Samuel P, Dash et al. Japanese encephalitis in India: An overview. Indian J. Pediatrics 2004; 71: 609-615. 23)

7. Glaser CA, Gilliam S, Schnuer D, Forghani B, Honarmand S, Khetsuriani Wet al. In search of encephalitis etiologies: diagnostic challenges in the California Encephalitis Project; 1998-2000. Clin.Infect.Dis. 2003; 36: 731-742.

8. Pallansch MA, Roos RP, Enteroviruses. Polioviruses, coxsackie viruses, echoviruses, and newer enteroviruses. In Fields Virology 4th edition, Edited by: KnippeDM, Howley PM, Philadelphia, PA: Lippincott Williams and Wilkins 2001;723-775.

9. Etiology of acute encephalitis, available at www.ajtmh.org/content/66/2/200fullpdf, access on 15/07/2014.

10. Touch S1, Grundy J, Hills S, Rani M, Samnang C, Khalakdina A, Jacobson $\mathrm{J}$. The rationale for integrated childhood meningoencephalitis surveillance: a case study from Cambodia. Bull World Health Organ. 2009 Apr;87(4):320-4.

11. Schuchat A, Robinson K, Wenger JD, et al. Bacterial meningitis in the United States in 1995. Active Surveillance Team. N Eng J Med.1997; 337:970.

12. Jayaraman Y, Veeraraghavan B, Chethrapilly Purushothaman GK, Sukumar B, Kangusamy B, Nair Kapoor A et al. Hospital Based Sentinel
Surveillance of Bacterial Meningitis (HBSSBM) Network Team. Burden of bacterial meningitis in India: Preliminary data from a hospital based sentinel surveillance network. PLoS One. 2018 May 16;13(5):e0197198. doi: 10.1371/journal.pone.0197198. PMID: 29768458; PMCID: PMC5955554.

13. Leimkugel, J \& Racloz, Vanessa \& Silva, L \& Pluschke, Gerd. (2009). Global review of meningococcal disease. A shifting etiology. Journal of Bacteriology Research. 1. 6-18.

14. S. Selim, Mohamed A. El-Barrawy, Magda E. Rakha, Samuel L. Yingst, Magda F. Baskharoun. "Microbial Study of Meningitis and Encephalitis Cases'. J Egypt public health association 2007;82(1-2)1-19.

15. Ajit Rayamajhi, Imran Ansari, ,Elizabeth Ledger, Krishna P. Clinical and prognostic features among children with acute encephalitis syndrome in Nepal; A retrospective study. BMC Infect Dis. 2011; 11: 294-297.

16. Joseph L Melnick. Enteroviruses. Polioviruses, Coxsackie viruses, echoviruses and newer enteroviruses; Field's virology 3rdedition. Lippincott-Raven Publishers, Philadelphia.1996:p.655-682.

17. Mark J Abjung. Nelson's Textbook of pediatrics 17TH ed.Sauunders Publ .2004;1043-1048.

18. C. A. Glaser,1 S. Honarmand,1 L. J. Anderson,et alBeyond Viruses: Clinical Profiles and Etiologies Associated with Encephalitis ,Clinical Infectious Diseases 2006; 43:1565-77.

Copyright: (C) the author(s), 2019. It is an open-access article distributed under the terms of the Creative Commons Attribution License (CC BY 4.0), which permits authors to retain ownership of the copyright for their content, and allow anyone to download, reuse, reprint, modify, distribute and/or copy the content as long as the original authors and source are cited.

How to cite this article: Bhardwaj P, Tripathi R. Magnitude of Bacteriological Etiology in Acute Encephalitis Syndrome: A Cross-Sectional Study. Asian J. Clin. Pediatr. Neonatol.2019;7(4):05-13.

DOI: dx.doi.org/10.21276/ajcpn.2019.7.4.2

Source of Support: Nil, Conflict of Interest: None declared. 